\title{
How to Use Equipment to Measure the Analog Signal by Means of FPGA System
}

\author{
Oleksandr Vorgul \\ Department of Microprocessor Technologies and Systems (MTS) \\ Kharkiv National University of Radio Electronics \\ Kharkiv, Ukraine \\ oleksandr.vorgul@nure.ua
}

\begin{abstract}
This article is devoted to design of a measurement system based on specialized FPGA. A balance of ACD and DAC channels through output from one side and computation power of FPGA from another side is considered. Possibilities for obtaining one more tool for screening the signal processing is proposed
\end{abstract}

Keywords-FPGA, analog signal, digital signal processing, measurement system

\section{INTRODUCTION}

A modern developer should be able to create modern devices. Their scope can be very wide. This measurement and intelligent devices, and medical equipment, and even telecommunications with multimedia. Constantly something new appears and you need to be ready for it.

Specialists in this field are required in firms engaged in research and design ( $R \& D$ ) of equipment and embedded solutions of computing and measuring equipment, telecommunications, medicine, automotive, environmental protection, and so on.

Great opportunities for the developer provides software company Xilinx, specialized for chips manufactured by the same company. Through the efforts of the company, the software is constantly being improved in terms of efficiency and flexibility. For the FPGA of the 7th generation, the Vivado software package [1] is recommended for use. The possibilities are expanding at the expense of working at different logical levels: in addition to the traditional level of register transfers, the new version has the opportunity to work at the system level. The list of valid programming languages was updated accordingly: if at the level of register transfers it is VHDL or Verilog, then at the system level it is SystemC, C or Python [1,2].

The Vivado software package refers to end-to-end software systems. It allows you to design modern devices on chips manufactured by Xilinx.

This company today produces PLM (CPLD), FPGA (FPGA), FPGA with pre-installed processor cores (SoC), as well as custom integrated circuits (ASIC). These types of microcircuits are related to microcircuits of different degree of integration, differ in the homogeneity of the internal structure, internal resources and purpose.

So, the main structural unit of the PLM is a 4 or 6 input configurable logic unit (CLB) with the ability to buffer outputs, allowing to implement all sorts of logical functions.

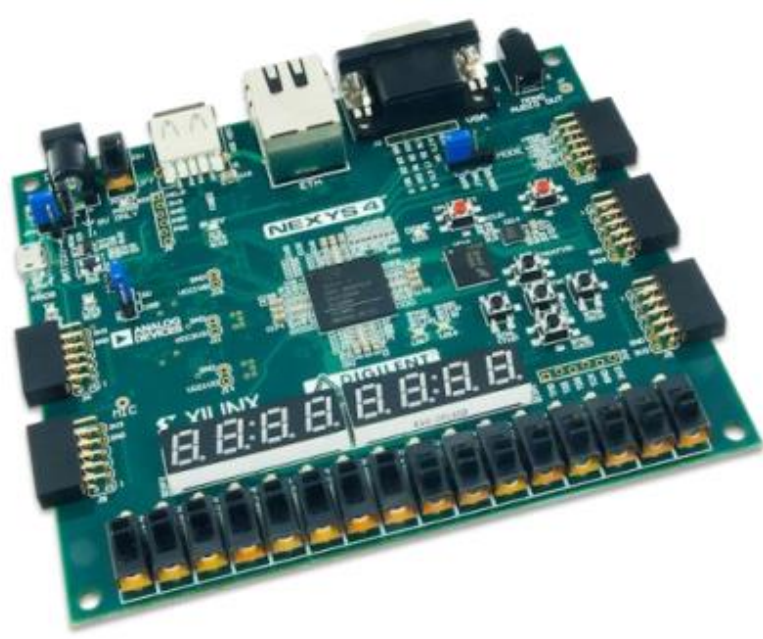

Fig. 1. Nexys 4 DDR with Artix-7 FPGA

FPGA 7 series differs from PLM not only in the fact that they have more such units on board, but also in the presence of specialized subsystems located in separate zones. For digital signal processing, this is a 12-bit ADC and a DSP48E1 digital processing unit (layer). If there is additional memory on the chip and the ability to connect via a wide high-speed channel of external memory, the capabilities of the PLM and FPGA differ significantly. The company produces a FPGA line optimized in terms of cost, price / quality ratio, efficiency (energy-performance ratio and speed) [3].

To implement algorithms that are demanding of computational resources, the company offers FPGAs with processors located on the same chip, which, from many points of view, simplifies system design.

All these innovations pose a task for software developers to maintain very diverse products. So, for PLA, apparently, is enough support for languages, such as VHDL or Verilog. Of course, a modeling module will be required taking into account time constraints and a modeling module for the implemented structure. Only now, to work with FPGAs, it will be necessary to work with a more complex system, with much larger amounts of memory and logical connections, with a large number of dedicated hardware adders and multipliers on board. And to work with multiple processors on board, you will need a language that allows you to operate 
with a higher level of abstractions than Verilog and VHDL allows.

In such conditions, we are faced with an exciting, but not an easy task to study the Xilinx product range and software, their capabilities, application features for creating equipment for various purposes.

\section{HOW TO MAKE A MEASUREMENT STUDIO OUT OF THIS}

Suppose we are going to measure an analog signal, using all the powers of new platform. We will need some analog inlet into digital system and maybe analog outlet for utilizing the result.

And here we go to work. We use FPGA when creating digital equipment for various purposes. And we implement some algorithm. What analog interfaces do manufacturers offer for use?

For the input analog signal onboard the Artix-7 platform, there is a 12-bit ADC with a conversion frequency of $1 \mathrm{MHz}$. Even for the youngest platform, this is not very much. PWM is proposed from the output channels for the analog signal, there is no DAC. Although the platform has enough ports to connect an external 12 or 16 bit D / A converter [5].

When performing various studies, it may be convenient to use the VGA monitor output as a graphical output device. This will require learning to perform the following operations.

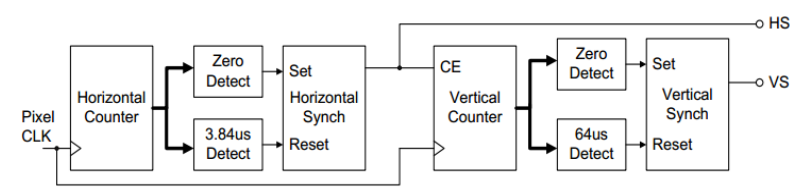

Fig. 2. VGA display controller block diagram

1. Display a digital stream (picture) on a monitor with given physical parameters: screen size in pixels and frequencies in rows and frames

2. Using the previous item as a standard procedure, display static graphic information (borders of graphic areas, scales, division prices, titles and labels on axes) and dynamic information (data displayed on charts)

3. The capabilities of the measuring complex will increase if you add the ability to interrogate hot keys that allow you to measure parameters and maintain cursor measurements

4. The data displayed on such a monitor can be of various types. The first thing that comes to mind is the dependence of the signal on time in real time, its spectral or correlation function.

The main problem is that the system should be balanced in its part to meet requirements for the task given. It is understood that a board like Nexys 4 DDR is designed as universal product for a broad circles of tasks and needs. It contains quite a lot of up-to-date digital interfaces like PCI-E
$10 \mathrm{GbE}$ to name a few. Maybe, it is a case when one wants something special?

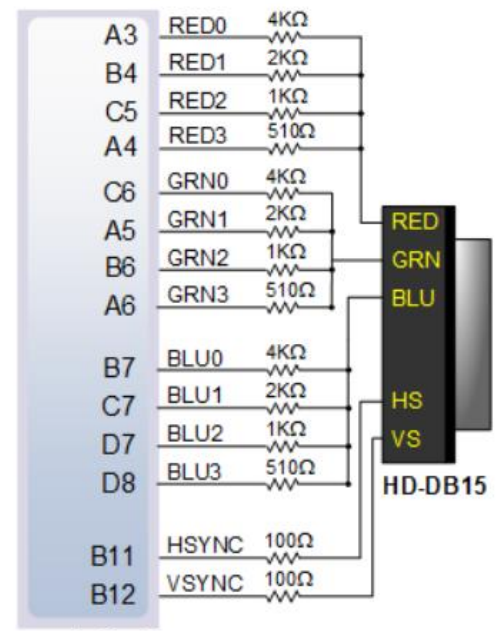

Artix-7

Fig. 3. VGA interface on Nexys 4 DDR

\section{PERSPECTIVES OF THE NEAREST STEPS}

If the aim is to design a DSP system with one or two analog inputs and one or two analog outputs, and suppose we try to process analog data with 12 bit ADC on moderate frequency, then all the power of Artix 7 platform with 100 $\mathrm{MHz}$ clock frequency and a little bit more will be quite handy. And maybe attaching couple of standalone ADCs and DACs with clock frequency close to $100 \mathrm{MHz}$ can improve the characteristics of our system.

Besides, VGA channel utilization can establish monitoring of the signal in different forms - temporal, spectral, correlation, wavelet.

\section{REFERENCES}

[1] Moiseev N.N. Mathematical problems of system analysis: [Proc. manual for universities on spec. "Apply Mathematics "] / N. N. Moiseev. - M .: Science, 1981. - 487.

[2] Digital signal processing and MATLAB: proc. manual / A.I. Solonina, D.M. Klionsky, T.V. Merkucheva, S.N. Perov. - SPb .. BHV-Petersburg, 2013. - 512 pp., Ill . - (Educational literature for universities).

[3] V. Semenets. Designing digital systems using the VHDL language: studies. manual / V.V. Semenets, I.V. Khakhanova, V.I. Khakhanov; MES of Ukraine, KNURE. - Kharkov: KNURE, 2003. - 492 p.

[4] Semenets V. V. Technology of electronic equipment interconnections: studies. for universities / V. V. Semenets, John Kratz, I. Sh. Nevludov, V. A. Palagin. - H.: ed. SMITH, 2005. - 432 p. [Semenets V. V., Kratts J., Nevlyudov I. Sh., Palagin V. A].

[5] Nexys4 ${ }^{\text {TM }}$ FPGA Board Reference Manual.

[6] S. Sakalo, V. Semenets and O. Azarhov, High frequencies in medicine (therapy and diagnostics): Teaching manual. Kharkov: KNURE; Collegium, 2005, p. 264.

[7] V. Semenets, "Technical aspects for development laboratory base for learning FPGA and microcontroller systems," 2009 10th International Conference - The Experience of Designing and Application of CAD Systems in Microelectronics, Lviv-Polyana, 2009, pp. 145-145. 